oder theoretisch gehaltvollen Begriffe auszuwählen und in einen Zusammenhang zu stellen und dafür die anderen Schlagwörter in den Orkus zu schicken oder sie dort in Frieden ruhen zu lassen. Einige Grundbegriffe, wie der der politischen Öffentlichkeit selbst und auch der PR-Begriff, bleiben unterreflektiert. Zumindest die hier kaum berücksichtigte amerikanische PR-Forschung um Larissa und James Grunig würde unter politischen Public Relations genau das verstehen, was Kamps im $\mathrm{Zi}$ tat oben als Kommunikationsmanagement bezeichnet.

Eine stärkere Strukturierung und Synthese der vielfältigen Befunde und Ansätze und auch ein Index hätten den wissenschaftlichen Gebrauchswert dieses Buches erhöht. Es ist als Fundus der Ergebnisse deutscher Forschung und der aktuellen Befindlichkeiten der politischen Kommunikation in Deutschland nützlich und verdienstvoll. Es bleibt zukünftiger Forschung vorbehalten, die Konturen einer oder mehrerer konkurrierender Theorien politischen Kommunikationsmanagements $\mathrm{zu}$ entwickeln. Gerade wenn es um grenzüberschreitende Phänomene wie das der Professionalisierung politischen Kommunikationsmanagements geht, verdient es auch die internationale Forschung, noch stärker berücksichtigt zu werden.

Michael Brüggemann

\section{Malthe Wolf}

\section{Ökonomische Erfolgsfaktoren privater Fernsehveranstalter}

Eine empirische Analyse externer und interner Erfolgsfaktoren

München: Reinhard Fischer Verlag, 2006. 382 S. (Reihe Medien-Skripten; Band 47)

ISBN 3-88927-410-2

Die Digitalisierung der Übertragungstechnik im Fernsehmarkt und die aktuell sinkenden Werbeerlöse resultieren in ein wachsendes Interesse an den strategischen Erfolgsfaktoren der privaten Fernsehbranche.

Im Rahmen einer empirischen Untersuchung widmet sich Malthe Wolf diesem Wandel. Im 10 Kapitel umfassenden Buch „Ökonomische Erfolgsfaktoren privater Fernsehveranstalter" fokussiert der Autor auf die wirtschaftswissenschaftliche Erfolgsfaktorenforschung in Rah- men der strategischen Medienmanagementforschung. In diesem Sinne definiert Wolf im einleitenden Kapitel die Zielsetzung der Arbeit als die theoretische Identifikation der relevanten Erfolgspotenziale für die private Fernsehbranche und deren empirischer Überprüfung.

Im zweiten Abschnitt erfolgt die Darstellung der deutschen Fernsehlandschaft durch die Abgrenzung der für die Untersuchung relevanten Märkte und ihrer Segmente. Der Fokus der Studie richtet sich dementsprechend im weiteren Verlauf auf die werbefinanzierten sowie entgeltfinanzierten Programme, während die gebührenfinanzierten, öffentlich-rechtlichen Sender als konstanter Marktfaktor angesehen werden. Im dritten Kapitel widmet er sich dem Status Quo der bisherigen Erfolgsfaktorenforschung, wobei klassische Konzepte einer näheren Beurteilung unterzogen werden. Im Ergebnis verwendet Wolf mehrere Elemente von vor allem neueren Forschungsansätzen zur Systematisierung von Methoden der Erfolgsfaktorenforschung für die Modellkonstruktion seiner Studie. Er nutzt einen explorativ-quantitativen Ansatz zur Datenerhebung, der von qualitativen Ansätzen unterstützt wird.

In Bezug auf die Ausrichtung am strategischen Management wird im vierten Kapitel die Erfolgsmessung als ein multidimensionales Konstrukt definiert. Konkret bedeutet dies, dass Erfolg als ein vierdimensionales Aggregat aus den Erfolgsdimensionen Rezipientenwert, Werbekundenwert, Marktanteil in der Zielgruppe und Umsatz interpretiert wird.

Gegenstand des fünften Kapitels ist die Analyse der vorhandenen medienökonomischen Literatur speziell zum Thema der Erfolgsfaktoren der Branche der privaten Fernsehveranstalter und die Umsetzung der bisher diskutierten Ansätze zur Modellbildung. Dabei erfolgt die Modellbildung dergestalt, dass der Aufbau des Modells in drei Bereiche unterteilt wird, die sich auf die verschiedenen Erfolgspotenzial-Quellen (Branche, Umwelt, Unternehmen) beziehen.

Erläuterungen zur Struktur, der empirischen Umsetzung und Durchführung der Studie schließen sich im nächsten Kapitel an: Das Erhebungsverfahren, das sich aus einer OnlineBefragung und einer schriftlichen Befragung zusammensetzt, richtet sich an eine vorläufige Grundgesamtheit von 362 privaten Fernsehveranstaltern, wobei als Auskunftspersonen Führungskräfte auf oberster Managementebe- 
ne herangezogen werden. Die Rücklaufquote beträgt knapp 27 Prozent, so dass der Untersuchung eine Stichprobe von 171 Personen zugrunde liegt. Die Auswertung der Ergebnisse erfolgt in zwei Schritten, wobei im siebten Kapitel in der ersten Phase die Ergebnisse der Befragung sowohl anhand univariater Auswertungen sowie mittels bivariater Gruppenvergleiche differenziert dargestellt werden. Vereinzelt wird auch die Standardabweichung in der Funktion eines Streuungsparameters zur Beurteilung der Homogenität innerhalb der Segmente (z. B. Spartenprogramm, Pay-TV) herangezogen.

Im achten Kapitel werden Faktorenanalysen zur Konstruktvalidierung eingesetzt. Die Analyse konzentriert sich dabei auf die internen Erfolgspotenziale. Um die Beziehung zwischen Erfolgsfaktoren und den erfolgsrelevanten Dimensionen in den Segmenten beurteilen zu können, werden einfache Regressionsanalysen eingesetzt. Bedingt durch die Anwendung dieser Verfahren muss der Untersuchungsbereich auf die zwei Gruppen der Free-TV-Anbieter (national und regional) beschränkt werden, da die anderen Segmente aufgrund der geringen Fallzahlen nicht dem Anforderungsprofil des Verfahrens genügen.

Insgesamt werden die Erfolgsfaktoren in fünf Regressionsanalysen mit den erfolgsrelevanten Dimensionen in Beziehung gesetzt, wobei als weitere erfolgswirksame Größe die Produktqualität zusätzlich $\mathrm{zu}$ den theoretisch bestimmten Dimensionen in Kapitel 4 empirisch bestätigt werden konnte. Für die Branche „Free-TV national“ entstammen die meisten erklärenden Erfolgsfaktoren dem Bereich der Steuerung und Planung. Hierbei generiert die Dimension des Werbekundenwerts mit stark signifikanten Einflussvariablen wie "Programmangebot, Crossmedia und Image“ das höchste Bestimmtheitsmaß, betrachtet man den Einfluss der Erfolgsfaktoren über alle Erfolgsmaße hinweg, so kann dem Faktor ,Wettbewerbsposition" am häufigsten ein Einfluss nachgewiesen werden.

Für die Branche „Free-TV regional“ kann ebenfalls die Erfolgsdimension Werbekundenwert den größten Anteil der Varianz mit dem hochsignifikanten Einfluss des Erfolgsfaktors „strategische Planung“ erklären. Als häufigster Faktor tritt in dieser Gruppe die „Wiederholungsrate von Formaten " auf.

Eine Diskussion der Ergebnisse gefolgt von
Implikationen für die Forschung und das $\mathrm{Ma}-$ nagement rundet das Buch ab.

Insgesamt ist das Buch, das zugleich eine Dissertation an der Universität Hohenheim darstellt, interessant zu lesen und gut nachzuvollziehen. Allerdings liegen kleinere methodische Schwächen vor. So weisen beispielsweise negative Cronbach's Alpha-Werte auf falsche Kodierungen hin. Zudem wählt der Autor eine reflektive Messung der Indikatoren, die aber besser formativ gemessen werden sollten, wenn es um die Identifikation von Erfolgsfaktoren geht. Dennoch, das Buch gibt einen sehr schönen Überblick über die Industrie und liefert durch die zahlreichen deskriptiven Ergebnisse und theoretischen Analysen einen deutlichen Mehrwert. Entsprechend ist das Buch nicht nur Wissenschaftlern, sondern auch TVManagern zu empfehlen.

Michel Clement

\section{Andreas Ettenhuber}

\section{Die Beschleunigung des Fernsehverhaltens}

Sekundäranalyse von Daten aus dem GfKFernsehpanel

München: Fischer, 2007. - 188 S.

(Reihe Rezeptionsforschung; 9)

ISBN 978-3-88927-426-7

Die Beschleunigungstendenzen gesellschaftlicher Prozesse und von Alltagshandeln sind übergreifende Schlüsselthemen moderner Gesellschaften. In Kombination mit der Mediennutzung ergibt sich ein spannendes Thema, das Andreas Ettenhuber in seiner Publikation aufgegriffen hat. Mit der langfristigen Untersuchung „Die Beschleunigung des Fernsehverhaltens" auf Basis der Daten aus dem AGF/GfK-Fernsehpanel schafft er selbst einen Kontrapunkt zu dem auch in der empirischen Forschung um sich greifenden Phänomen der Beschleunigung und Hektik der Verbreitung empirischer Befunde in unserer Gesellschaft.

Auf Basis umfangreicher Literatur beschreibt er systematisch unterschiedliche Forschungsansätze, die sich mit dem Phänomen der Beschleunigung gesellschaftlicher Phänomene und insbesondere der Fernsehnutzung beschäftigen. Die Unterscheidung zwischen Effekten der Binnenstruktur von Sendungen, der Struktur des Programmablaufs sowie des aktiven Handelns der Fernsehzuschauer sind 